\title{
Congenitally transmitted Chagas disease in Canada: a family cluster
}

\author{
Pierre J. Plourde MD, Kamran Kadkhoda PhD D(ABMM), Momar Ndao DVM PhD
}

Cite as: CMAJ 2017 December 4;189:E1489-92. doi: 10.1503/cmaj.170648

\section{Case 1}

A 34-year-old, previously healthy woman presented to her family physician with fatigue. She astutely requested testing for Chagas disease, knowing her mother had previously been given this diagnosis. She was otherwise asymptomatic, with no evidence of intestinal obstruction or constipation, and no heart failure or arrhythmia. Physical examination and routine biochemistry, hematology, renal and hepatic blood tests were normal. A 12-lead electrocardiogram (ECG) was normal. A polymerase chain reaction assay for Trypanosoma cruzi sent to the National Reference Centre for Parasitology in Montréal was positive. Based on a diagnosis of indeterminate Chagas disease (chronic asymptomatic infection), she started treatment with benznidazole. However, she developed a severe, diffuse drug rash nine days into treatment and was forced to stop the treatment. She declined treatment with nifurtimox. She remains asymptomatic and has agreed to yearly ECGs.

The patient was born in Canada and spent brief periods of time in Paraguay as a child, visiting relatives at the ages of 5 and 10 years for one to two months only. On both trips, she stayed in well-screened rural concrete housing. There was no other travel to Chagas-endemic countries. Medical history was unremarkable, with no history of blood transfusions. Family history showed that in addition to her mother, her younger brother had also been given a diagnosis of Chagas disease. Her children (ages 2, 4 and $7 \mathrm{yr}$ ) all tested negative for Chagas disease.

\section{Case 2}

The patient's younger brother (age $31 \mathrm{yr}$ ) reported donating blood for many years until 2010, when Canadian Blood Services discovered that he had positive Chagas antibodies, once riskbased donor testing was implemented. He was asymptomatic. He was born in Canada and had also visited relatives in Paraguay with his sister, at the ages of two and seven years. He had no history of blood transfusions. Physical examination, routine blood tests and a 12-lead ECG were normal. He had a positive polymerase chain reaction assay for $T$. cruzi (performed at the

\section{KEY POINTS}

- Immigrants from countries with higher endemicity for Chagas disease (including Argentina, Bolivia, El Salvador, Guatemala, Honduras, Panama and Paraguay) should be offered testing.

- All offspring born of women diagnosed with Chagas disease should be offered testing for the condition.

- The earlier a diagnosis of Chagas disease is made and the younger a person is when treatment is given, the more likely treatment will be beneficial.

- The management of Chagas disease requires lifelong evaluation and follow-up.

National Reference Centre for Parasitology). He declined treatment for indeterminate Chagas disease but agreed to follow-up with yearly ECGs.

\section{Case 3}

The patient's older sister ( $41 \mathrm{yr}$ ) subsequently presented for testing and was found to be positive for T. cruzi antibodies. She was also born in Canada, asymptomatic, and briefly visited relatives in Paraguay only once at the age of 11 years. She had no history of blood transfusions. Physical examination, routine blood tests and a 12-lead ECG were normal. She had a positive polymerase chain reaction assay for T. cruzi (performed at the National Reference Centre for Parasitology). She also declined treatment and agreed to follow-up with yearly ECGs. Her children (aged 2, 4, and $6 \mathrm{yr}$ ) tested negative for Chagas disease.

The mother of these siblings was born in Paraguay in 1949. She moved to Bolivia and lived there from 1958 to 1972 . She immigrated to Canada in 1972, where her four children were born. She was given a diagnosis of asymptomatic Chagas disease on a visit to Paraguay in 2012 and, upon returning to Canada, had confirmatory positive polymerase chain reaction testing for T. cruzi (performed at the National Reference Centre for Parasitology). She was not offered treatment because of her advanced age and lack of clinical signs. Her other daughter is also asymptomatic and has tested negative for T. Cruzi antibodies. 


\section{Discussion}

Congenitally acquired Chagas disease has rarely been reported in Canada. ${ }^{1,2}$ Although it is difficult to confirm, we hypothesized that these three adults with polymerase chain reaction-positive, indeterminate Chagas disease acquired their infection congenitally, as they were born in Canada, spent small periods of time in an endemic area (fewer than three months cumulatively) and were born to a mother who was parasitemic and never treated for Chagas disease.

Chagas disease, caused by the protozoan parasite Trypanosoma cruzi, is spread primarily by triatomine insects by direct inoculation of infected vector feces into a bite wound or via intact mucous membrane contact ${ }^{3}$ (Figure 1). Vector-borne transmission occurs in large areas of South and Central America and southern regions of North America (including the southern United States) ${ }^{3}$ (Figure 2). An estimated 5 to 10 million people are infected in Latin America and the US, with a projected estimate that 200000 infected persons will die over the next five years owing to complications from heart disease related to Chagas disease. ${ }^{4}$

Chagas disease is also spread via blood transfusion and organ transplantation, from mother to fetus, and by oral ingestion of contaminated juice or water. ${ }^{1,3,5,6}$ Congenitally acquired Chagas disease occurs in only $5 \%$ of at-risk newborns and is associated with younger maternal age, higher parasitemia and HIV infection. . $^{5}$
As Chagas disease is not a notifiable communicable disease in Canada, there are little data on the number of undiagnosed, untreated cases. With increasing immigration from endemic countries, it is estimated that thousands of individuals with undetected T. cruzi infection live in Canada. ${ }^{8}$ Vertical transmission in endemic countries is estimated to occur at rates of $5 \%-6 \% .^{5}$ In mothers who are parasitemic, vertical transmission is as high as $31 \% .^{7}$ Based on prevalence rates of Chagas disease in the maternal country of origin and an average $5 \%$ risk of vertical transmission, one can estimate that from thousands of undiagnosed immigrant mothers, there could be up to 100 to 200 undiagnosed and untreated congenitally infected persons in Canada.

\section{Clinical presentation}

After an incubation period of one to two weeks, about $5 \%$ of those infected will develop nonspecific symptoms, such as fatigue, anorexia and fever; the rest will be asymptomatic in the acute phase. ${ }^{3}$ The classically described signs of an erythematous, indurated lesion at the site of entry of the parasite (a chagoma) and the painless periorbital swelling (Romana sign) are uncommon. Acute symptomatic presentation is more common in children and older people.

Congenital Chagas disease is usually asymptomatic or presents with mild nonspecific symptoms, including low birth weight, low Apgar scores, hepatosplenomegaly, anemia or thrombocytopenia. ${ }^{5}$ More serious but uncommon presentations associated with high

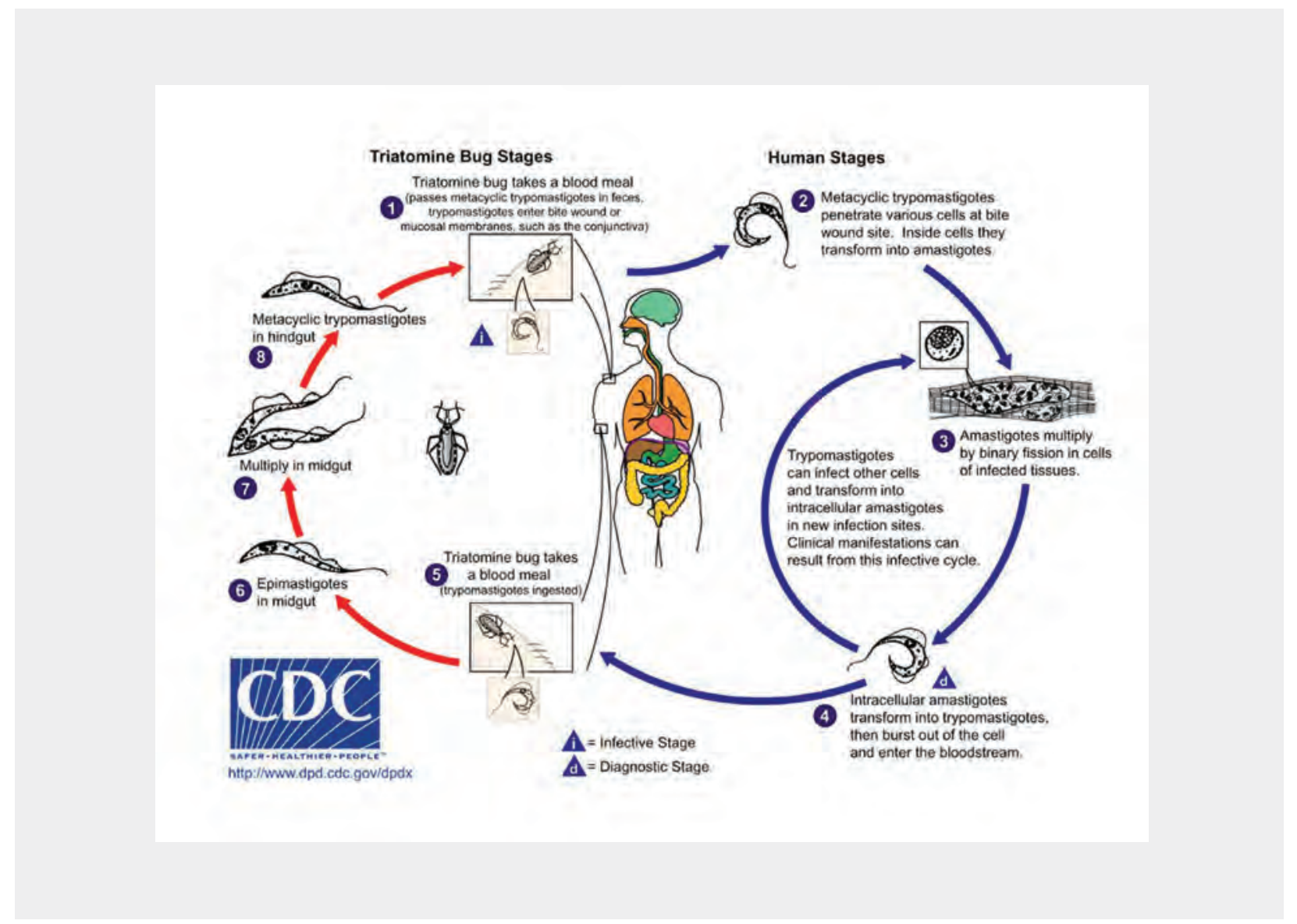

Figure 1: Life cycle of Trypanosoma cruzi (reprinted with permission from the US Centers for Disease Control and Prevention, Atlanta). 
mortality include myocarditis, meningoencephalitis and respiratory distress. ${ }^{5,9}$ Of those without symptoms at birth, $70 \%-80 \%$ will progress to a chronic indeterminate form of the disease, with $20 \%$ $30 \%$ progressing to symptomatic disease over several years to decades: primarily cardiac and, less frequently, gastrointestinal. ${ }^{9}$

Individuals with untreated chronic asymptomatic or indeterminate Chagas disease (as with the three patients described here) are at risk of severe reactivation in the setting of immunosuppression. ${ }^{3}$ Severe reactivation can manifest as potentially fatal acute myocarditis, congestive heart failure, meningoencephalitis and febrile neutropenia following chemotherapy. ${ }^{3}$ About 20\%-30\% of untreated individuals progress to chronic infection, with the parasite invading smooth muscle, leading to cardiomegaly with arrhythmias and cardiac failure, megacolon or megaesophagus. ${ }^{3}$

\section{Diagnosis}

Given that Chagas disease is most often asymptomatic, it is best diagnosed using a high index of suspicion for screening those most at risk of infection, including immigrants from endemic countries (especially Argentina, Bolivia, El Salvador, Guatemala, Honduras, Panama and Paraguay $)^{8}$ and offspring born to infected mothers. These three cases were discovered somewhat incidentally: one owing to a request for testing, one to successful Canadian Blood Services blood screening, and the other because two siblings tested positive. Had the first patient not made the request for testing and the second not donated blood, none would have received the diagnosis even though all three were aware they had been born to a mother with parasitemic Chagas disease. Fortunately, none of the children born to the infected daughters tested positive.

Chagas disease is diagnosed by $T$. cruzi antibody detection or by polymerase chain reaction assay, both performed at the National Reference Centre for Parasitology at McGill University in Montréal. Serologic detection of Chagas disease suggests previous infection, whereas the polymerase chain reaction assay is more specific for an active parasitemic infection and may be performed after treatment to ascertain microbiologic cure.

An initial workup for Chagas disease should include chest radiography and 12 -lead ECG to rule out presymptomatic cardiac

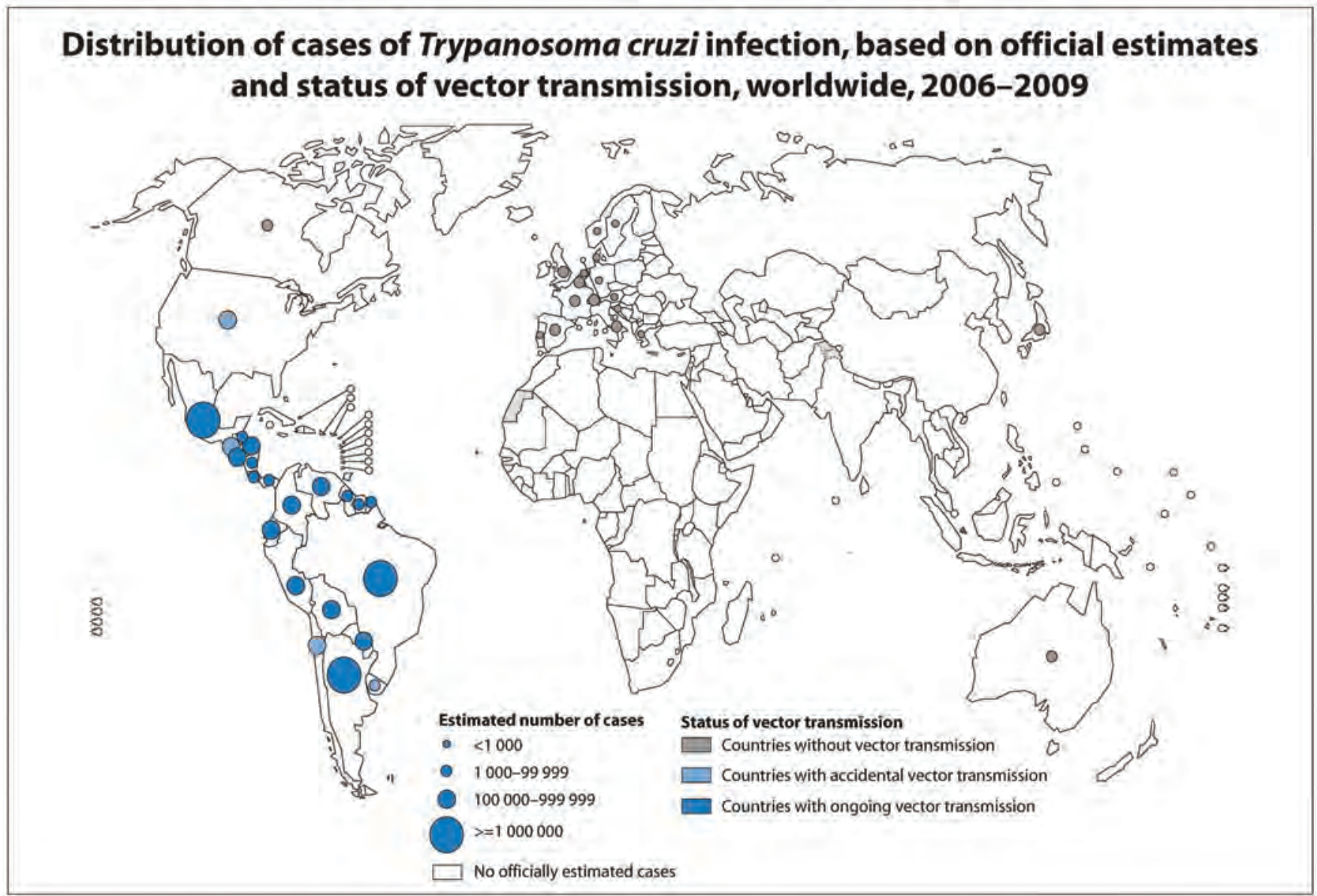

The boundaries and names shown and the designations used on this map do not imply the expression of any opinion whatsoever on the part of the World Health Organization concerning the legal status of any country, territory, city or area or of its authorities, or concerning the delimitation of its frontiers or boundaries. Dotted lines on maps represent approximate border lines for which there may not

Data Source: World Health Organization Map Production: Control of Neglected Tropical Diseases (NTD) World Health Organization

yet be full agreement. 0 WHO 2010 . All rights reserved

Figure 2: Distribution of cases of Trypanosoma cruzi infection based on official estimates and status of vector transmission, 2006-2009 (reprinted with permission from the World Health Organization; accessed at http://gamapserver.who.int/mapLibrary/Files/Maps/Global_chagas_2009.png) (accessed 2017 Aug. 1). 
rhythm disturbances and cardiomegaly. Baseline complete blood count and liver enzymes may be needed if treatment is being started. If cardiac symptoms are present, echocardiogram, 24-hour Holter monitoring, exercise stress test and cardiology consultation are suggested. With gastrointestinal symptoms, barium swallow, barium enema and gastroenterology consultation are suggested. ${ }^{3,9}$

\section{Treatment and long-term follow-up}

Based on treatment efficacy data, treatment is generally suggested for everyone with acute Chagas disease, congenital Chagas disease in newborns, reactivation Chagas disease with HIV coinfection or immunosuppression, and all children 18 years and under with any stage of Chagas disease..$^{3,9}$ In addition, some experts also suggest treating adults aged 19-50 years with indeterminate Chagas disease or early cardiomyopathy, based on limited efficacy data; and women of reproductive age, based on data showing that women treated before pregnancy are significantly less likely to transmit $T$. cruzi to their offspring. ${ }^{3}$ Persons with indeterminate Chagas disease older than 50 years do not benefit from treatment, nor do those with advanced irreversible

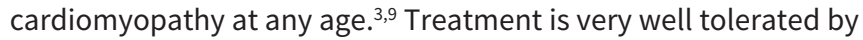
infants, with the best cure rates. Treatment of Chagas disease is more beneficial the earlier a diagnosis is made. Complete cures are higher in younger people, with the highest cure rates of more than $90 \%$ in infants who are treated during the first year of life. ${ }^{3,9}$

Treatment with benznidazole or nifurtimox can alleviate symptoms, clear the parasite and moderate the risk of future adverse outcomes. ${ }^{3,9}$ Treatment of chronic Chagas disease is controversial but is believed to be of benefit only in people younger than 50 years. ${ }^{3}$ People older than 50 years or with advanced cardiac disease are unlikely to benefit from treatment., ${ }^{9,10}$

Although most experts suggest treatment for people younger than 50 years without evidence of cardiac disease, in our case, all three siblings were unable or unwilling to complete treatment. Treatment with benznidazole or nifurtimox is associated with serious adverse effects, including neutropenia, thrombocytopenia and peripheral neuropathy (both drugs), severe photodermatitis (benznidazole) and substantial gastrointestinal intolerance (nifurtimox). ${ }^{9}$ Access to treatment is difficult in Canada. Both nifurtimox and benznidazole are intermittently available, and both can be obtained only through the Health Canada Special
Access Programme for nonmarketed drugs (www.hc-sc.gc.ca/ dhp-mps/acces/drugs-drogues/index-eng.php). Consultation with a specialist in infectious diseases or tropical medicine is suggested for anyone requiring treatment for Chagas disease.

Regardless of treatment, the management of Chagas disease also requires lifelong evaluation for its most common complications, including cardiac conduction anomalies, megaesophagus and megacolon. Minimally, experts suggest performing electrocardiography annually. ${ }^{3,9}$

Since Canadian Blood Services and Héma-Québec began screening for Chagas disease less than a decade ago, an increasing number of people with newly diagnosed Chagas disease have been identified. ${ }^{1,2}$ Given the substantial numbers of Latin American immigrants from endemic countries, there are likely thousands of undiagnosed, chronically infected persons with Chagas disease in Canada, ${ }^{8}$ with only a small minority discovered via blood donation. With large numbers of undiagnosed, foreignborn Canadians and the risk of congenital transmission through multiple generations, a high index of suspicion is needed for screening of Chagas disease in asymptomatic immigrants from Chagas disease-endemic countries, including screening offspring born of women who have received a diagnosis of Chagas disease.

\section{References}

1. Fearon MA, Scalia V, Huang M, et al. A case of vertical transmission of Chagas disease contracted via blood transfusion in Canada. Can J Infect Dis Med Microbiol 2013;24:32-4.

2. Mongeau-Martin G, Ndao M, Libman M, et al. A family cluster of Chagas disease detected through selective screening of blood donors: a case report and brief review. Can J Infect Dis Med Microbiol 2015;26:157-61.

3. Bern C. Chagas' disease. N Engl J Med 2015;373:456-66.

4. Pecoul B, Batista C, Stobbaerts E, et al. The BENEFIT trial: Where do we go from here? PLoS Negl Trop Dis 2016;10:e0004343.

5. Torrico F, Alonso-Vega C, Suarez E, et al. Maternal Trypanosoma cruzi infection, pregnancy outcome, morbidity, and mortality of congenitally infected and noninfected newborns in Bolivia. Am J Trop Med Hyg 2004;70:201-9.

6. Shikanai-Yasuda MA, Carvalho NB. Oral transmission of Chagas disease. Clin Infect Dis 2012;54:845-52.

7. Murcia L, Carrilero B, Munoz-Davila MJ, et al. Risk factors and primary prevention of congenital Chagas disease in a nonendemic country. Clin Infect Dis 2013;56:496-502.

8. Schmunis GA, Yadon ZE. Chagas disease: A Latin American health problem becoming a world health problem. Acta Trop 2010;115:14-21.

9. Bern C, Montgomery SP, Herwaldt BL, et al. Evaluation and treatment of Chagas' disease in the United States: a systematic review. JAMA 2007;298:2171-81.

10. Morillo CA, Marin-Neto JA, Avezum A, et al. BENEFIT Investigators. Randomized trial of benznidazole for chronic Chagas' cardiomyopathy. N Engl J Med 2015; 373:1295-306.

\section{Competing interests: None declared.}

This article has been peer reviewed.

The authors have obtained patient consent.

Affiliations: Winnipeg Regional Health Authority (Plourde), Travel Health and Tropical Medicine Services; Departments of Medical Microbiology and Infectious Diseases (Plourde, Kadkhoda), and Immunology (Kadkhoda), Rady Faculty of Health Sciences, University of Manitoba; Cadham Provincial Laboratory (Kadkhoda), Winnipeg, Man.; National Reference Centre for Parasitology (Ndao), Research Institute of the McGill University Health Centre, McGill University, Montréal, Que.

Contributors: All of the authors contributed to the conception and design of the work, drafted the manuscript, revised it critically for important intellectual content, gave final approval of the version to be published and agreed to be accountable for all aspects of the work.

Acknowledgements: The authors wish to thank the three siblings and mother who graciously participated in the preparation of this case report. The authors also thank the
Foundation of the McGill University Health Centre, and Henry Bishop at the US Centers for Disease Control and Prevention Division of Parasitic Diseases and Malaria for providing the life cycle figure. The National Reference Centre for Parasitology is supported by Public Health Agency of Canada/National Microbiology Laboratory grant number WPG6-39147 (005), the Foundation of the Montréal General Hospital and the Research Institute of the McGill University Health.

Correspondence to: Pierre Plourde, pplourde@wrha.mb.ca 